\title{
Metales pesados en truchas arcoíris (Oncorhynchus mykiss) de crianza intensiva de la zona noroeste del lago Titicaca
}

\author{
Heavy metals in intensive farmed rainbow trout (Oncorhynchus mykiss) from \\ northwestern Titicaca Lake
}

\author{
Heber N. Chui ${ }^{1}$, Bernardo Roque ${ }^{2}$, Edilberto Huaquisto', Danitza L. Sardón', \\ German Belizario $^{2}$, Alfredo P. Calatayud ${ }^{1}$
}

\section{Resumen}

Los sedimentos del lago Titicaca contienen metales pesados de origen minero, sugiriendo la contaminación de las granjas de peces que se desarrollan en sus aguas. El presente estudio tuvo como objetivo evaluar la concentración de metales pesados en los músculos de truchas arcoíris (Oncorhynchus mykiss) criadas en la zona noreste del lago Titicaca (3812 msnm), ubicado en las provincias de Puno y Huancané, Perú. Los metales pesados presentes en la carne de trucha se analizaron a través del método espectrofotométrico utilizando la técnica de plasma de acoplamiento inductivo junto a un espectrofotómetro de emisión óptica ICP-OES. La secuencia de la concentración de metales pesados hallada en los músculos de las truchas colectadas de la provincia de Puno fue: $\mathrm{Zn}>\mathrm{Fe}>\mathrm{Cd}>\mathrm{Mn}>\mathrm{Pb}>\mathrm{Cu}>\mathrm{Hg}$ y en la provincia de Huancané fue: $\mathrm{Zn}>\mathrm{Fe}>\mathrm{Mn}$ $>\mathrm{Cu}>\mathrm{Pb}>\mathrm{Cd}>\mathrm{Hg}$. Las concentraciones halladas no superaron los límites máximos permisibles recomendados por $\mathrm{WHO} / \mathrm{FAO}$, por lo que las truchas de las zonas evaluadas son aptas para el consumo humano.

Palabras clave: metales pesados, oncorhynchus mykiss, plasma de acoplamiento inductivo, truchas

\footnotetext{
${ }^{1}$ Instituto de Ciencias Naturales, Universidad Nacional del Altiplano de Puno, Perú

${ }^{2}$ Centro de Investigación Fundo Carolina, Universidad Nacional del Altiplano de Puno, Perú

${ }^{3}$ E-mail: hchui@unap.edu.pe; https://orcid.org/0000-0001-8869-9423
}

Recibido:17 de septiembre de 2020

Aceptado para publicación: 2 de abril de 2021

Publicado: 23 de junio de 2021

CLos autores. Este artículo es publicado por la Rev Inv Vet Perú de la Facultad de Medicina Veterinaria, Universidad Nacional Mayor de San Marcos. Este es un artículo de acceso abierto, distribuido bajo los términos de la licencia Creative Commons Atribución 4.0 Internacional (CC BY 4.0) [https:// creativecommons.org/licenses/by/4.0/deed.es] que permite el uso, distribución y reproducción en cualquier medio, siempre que la obra original sea debidamente citada de su fuente original 


\section{Abstract}

The sediments of Lake Titicaca contain heavy metals of mining origin, suggesting the contamination of the fish farms that develop in its waters. The present study aimed to evaluate the concentration of heavy metals in the muscles of rainbow trout (Oncorhynchus mykiss) raised in the northeast area of Lake Titicaca, at an altitude of $3812 \mathrm{~m}$, located in the provinces of Puno and Huancané, Peru. The heavy metals present in the trout meat were analyzed through the spectrophotometric method using the inductively coupled plasma technique together with an ICP-OES optical emission spectrophotometer. The sequence of the concentration of heavy metals found in the muscles of the trout collected from the province of Puno was $\mathrm{Zn}>\mathrm{Fe}>\mathrm{Cd}>\mathrm{Mn}>\mathrm{Pb}>\mathrm{Cu}>\mathrm{Hg}$ and in the province of Huancané was $\mathrm{Zn}>\mathrm{Fe}>\mathrm{Mn}>\mathrm{Cu}>\mathrm{Pb}>\mathrm{Cd}>\mathrm{Hg}$. The concentrations found did not exceed the maximum permissible limits recommended by WHO/FAO, so the trout from the evaluated areas are suitable for human consumption.

Key words: essential chemical elements, oncorhynchus mykiss, inductively coupled plasma

\section{INTRODUCCIÓN}

El pescado es un alimento de importancia por su aporte de nutrientes, tales como proteínas, ácidos grasos esenciales, minerales y vitaminas (Mohanty et al., 2018), por lo que es un complemento ideal de la dieta humana (Thilsted et al., 2016; Balami et al., 2019), especialmente para la salud cardíaca, por su elevado contenido de ácidos grasos $\omega$ 3 que incluyen los ácidos eicosapentaenoico (EPA) y docosahexaenoico (DHA) (Durmus, 2019). El consumo de pescado es recomendado para mantener alejado al cardiólogo (Peter et al., 2013), y sobre todo para la alimentación de la población de bajos recursos económicos (Kwasek et al., 2020).

La trucha arcoíris (Oncorhynchus mikkis), desde su introducción en el lago Titicaca en 1942, fue una alternativa importante que impulsó la pesca artesanal y comercial, lográndose capturar hasta $500 \mathrm{t}$ en 1965, disminuyendo posteriormente por la elevada presión de pesca (Everett, 1973). A par- tir de 1970, la crianza de truchas se convirtió en una actividad económica emergente, con un crecimiento sistemático (Araníbar et al., 2013), a través de una producción intensiva, registrando 1206 ten 2002 (FAO, 2005), 8543 t en 2009 (Chura y Mollocondo, 2009) y una producción que supera las 20000 toneladas para la comercialización con destino a los mercados de la república de Bolivia (Onda Azul, 2020).

El lago Titicaca es el recurso hídrico más importante de la meseta andina de Perú y Bolivia, donde desembocan los ríos procedentes de las partes altas, arrastrando metales pesados de origen minero que explotan oro y plata (Martínez et al., 2007). Los reportes indican que las aguas superficiales que escurren de las mineras están fuertemente contaminadas por drenaje ácido (Salvarredy et al., 2008), por lo que los sedimentos y la bahía interior del lago Titicaca están contaminados con metales pesados de origen minero (Cáceres et al., 2013; Moreno et al., 2017). SEs así que se ha encontrado mercurio en músculo (Gammons et al., 2006) y 
metales pesados $(\mathrm{Hg}, \mathrm{Cd}$ y $\mathrm{Pb})$ en hígado y músculos de los peces de vida libre de este lago, en niveles que superan los umbrales de seguridad establecidos (Monroy et al., 2014). Esta situación es preocupante, puesto que las truchas criadas en jaulas podrían estar también contaminadas por metales pesados pudiendo afectar la salud de la población que las consumen (Maurya et al., 2019).

Los metales pesados y metaloides más peligrosos para el medio ambiente incluyen $\mathrm{Cr}, \mathrm{Ni}, \mathrm{Cu}, \mathrm{Zn}, \mathrm{Cd}, \mathrm{Pb}, \mathrm{Hg}$ y As (Ali et al., 2019), siendo más importantes para el medio ambiente acuático el $\mathrm{Hg}, \mathrm{Cd}, \mathrm{Cu}, \mathrm{Pb}$ y $\mathrm{Zn}$, por lo que los peces se utilizan como bioindicadores de los efectos de la contaminación (Authman et al., 2015). De estos, $\mathrm{Pb}$, $\mathrm{As}, \mathrm{Cd}, \mathrm{Cu}$ y $\mathrm{Zn}$ son los más tóxicos para el humano (Huseen y Mohammed, 2019), con un índice de peligro para niños y adultos que desciende en el orden de $\mathrm{Cr}>\mathrm{As}>\mathrm{Pb}>\mathrm{Cd}$ $>\mathrm{Cu}>\mathrm{Ni}>\mathrm{Zn}$, con un riesgo cancerígeno de Cr y As, para niños y adultos, superiores a $10^{-4}$ (Wu et al., 2018). Es por esto que el monitoreo en los peces es muy importante a fin de garantizar el consumo de alimentos saludables (Authman et al., 2015; Bosch et al., 2016).

La bioacumulación de metales pesados en los peces y la distribución en sus órganos depende principalmente del ambiente acuático donde viven y sus hábitos de vida, debido a que los contaminantes metálicos no están uniformemente distribuidos en el medio ambiente acuático (Zhao et al., 2012). Existen grandes variaciones en las branquias, hígado y músculos, y una mayor acumulación de metales en la piel de los peces pelágicos (Canli y Atli, 2003), así como en intestinos y riñones (Rajeshkumar y Li, 2018). La mayoría de los estudios sobre acumulación de $\mathrm{Cu}, \mathrm{Zn}$ y Fe enfocan el hígado como el órgano objetivo; sin embargo, $\mathrm{Pb}$ y Mn presentan concentraciones más altas en las branquias, mientras que los músculos siempre muestran las concentraciones más bajas de todos los metales (El-Moselhy et al., 2014). Así mismo, las con- centraciones de metales más altas se encuentran en los peces omnívoros/herbívoros y las más bajas en los peces carnívoros (Bawuro et al., 2018).

Los estudios experimentales en truchas criadas en medios acuáticos con niveles crecientes de metales pesados han evidenciado que, esta especie acumula $\mathrm{Zn}$ y $\mathrm{Cu}$ en sus tejidos corporales en una relación directa con el incremento de las concentraciones de estos metales en el agua (Gündoðdu y Erdem, 2008). Así mismo, las truchas criadas en jaulas y alimentadas con alimentos peletizados acumulan en sus cuerpos $\mathrm{Hg}, \mathrm{Cd}$ y $\mathrm{Pb}$ (Majlesi et al., 2019), evidenciando que también son susceptibles a la contaminación por metales pesados. A partir de las consideraciones expuestas, el trabajo tuvo como objetivo evaluar las concentraciones de metales pesados ( $\mathrm{Pb}, \mathrm{Cd}, \mathrm{Hg}, \mathrm{Cu}, \mathrm{Fe}, \mathrm{Zn}$ y $\mathrm{Mn}$ ) en truchas arcoíris (Oncorhynchus mykiss) en dos áreas representativas de la zona noroeste del lago Titicaca de Perú, donde se practica la producción intensiva de esta especie acuícola con fines comerciales para la alimentación humana.

\section{Materiales y Métodos}

El estudio se realizó en las provincias de Puno y Huancané del departamento de Puno, Perú, donde se desarrolla la crianza intensiva de truchas arcoíris. Las muestras de truchas se obtuvieron de las jaulas flotantes de las localidades de Llachón (Puno) y Jonsani (Huancané), ubicadas en las coordenadas $15^{\circ} 43^{\prime} 34,4^{\prime \prime} \mathrm{S}, 69^{\circ} 47^{\prime} 27,1^{\prime \prime} \mathrm{O}$ y $15^{\circ}$ $17^{\prime} 41,2^{\prime \prime} \mathrm{S}, 69^{\circ} 43^{\prime} 49,0^{\prime \prime} \mathrm{O}$, respectivamente, ambas ubicadas a $3812 \mathrm{~m}$ de altitud en el lago Titicaca (Figura 1).

Las truchas fueron especímenes adultos, con talla y peso superiores a las de uso comercial (Cuadro 1), las mismas que fueron obtenidas con la asunción de que la acumulación de metales pesados en el cuerpo está en relación con la edad de los peces (Afshan et 


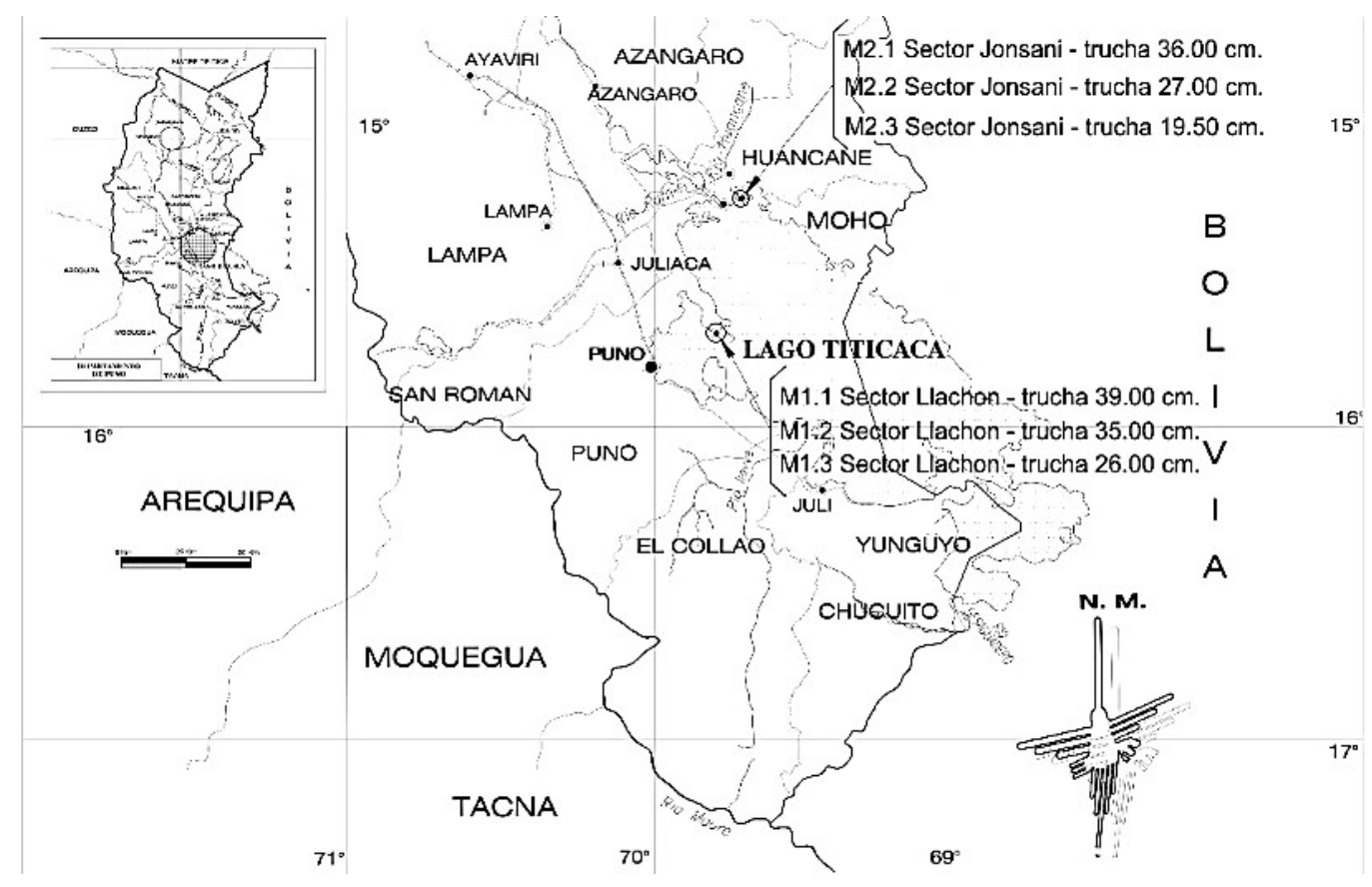

Figura 1. Ubicación geográfica de las zonas de muestreo de las truchas arcoíris (Oncorhynchus mykiss) en las provincias de Puno y Huancané (Perú)

al., 2014; Huseen y Mohammed, 2019) y que el monitoreo más importante constituye la carne por ser la parte comestible (Doan et al., 2020).

Los muestreos se realizaron en la época de lluvias (marzo), capturándose de las jaulas, al azar, nueve (9) truchas por zona, con pesos entre 700 y $820 \mathrm{~g}$ y tallas entre 21 y $34 \mathrm{~cm}$ de longitud. Los peces fueron procesados en fresco, obteniéndose los filetes de músculos sin piel, considerando que es la parte más comestible y de mayor valor comercial. Las muestras fueron envasadas en sobres de polietileno, congelados y enviados a los Laboratorios Analíticos del Sur de la ciudad de Arequipa, siguiendo estrictamente la cadena de custodia recomendada. El laboratorio se encuentra acreditado bajo la Norma NTP ISO/IEC 17025.2006.
Cuadro 1. Características de las truchas arcoíris (Oncorhynchus mykiss) en Puno y Huancané, Perú

\begin{tabular}{lcc}
\hline Variable & Puno & Huancané \\
\hline Talla, cm & $33.3 \pm 6.7$ & $27.5 \pm 8.3$ \\
Peso, $\mathrm{kg}$ & $0.81 \pm$ & $0.75 \pm 0.02$ \\
Edad, días & $485 \pm 35$ & $426 \pm 47$ \\
$\begin{array}{l}\text { Etapa de } \\
\text { desarrollo }\end{array}$ & Adulta & Adulta \\
\hline
\end{tabular}

El orden de la secuencia de concentración de metales pesados en la carne de las truchas colectadas en la provincia de Puno fue $\mathrm{Zn}>\mathrm{Fe}>\mathrm{Cd}>\mathrm{Mn}>\mathrm{Pb}>\mathrm{Cu}>\mathrm{Hg}$, 
Cuadro 2. Concentración de metales pesados $\left(\mathrm{mg} \cdot \mathrm{kg}^{-1}\right)$ en músculos de truchas arcoíris (Oncorhynchus mykiss) de crianza intensiva de dos zonas representativas del lago Titicaca de Perú ( $\mathrm{n}=9$ peces por zona)

\begin{tabular}{lcccccc}
\hline $\begin{array}{l}\text { Metales } \\
\text { pesados }\end{array}$ & Puno & Huancané & $\mathrm{P}_{(\alpha 0.05)}$ & $\begin{array}{c}\text { WHO/FAO } \\
(2015)\end{array}$ & $\begin{array}{c}\text { FSANZ } \\
(2013)\end{array}$ & $\begin{array}{c}\text { MHPRC } \\
(2013)\end{array}$ \\
\hline Plomo & $0.051 \pm 0.0028$ & $0.049 \pm 0.0032$ & 0.046 & 0.5 & 0.5 & 0.5 \\
Cadmio & $\mathrm{a}<0.022$ & $\mathrm{a}<0.022$ & - & $\mathrm{Nd}$ & $\mathrm{Nd}$ & 0.1 \\
Mercurio & $\mathrm{a}<0.0082$ & $\mathrm{a}<0.0082$ & - & $\mathrm{Nd}$ & 0.5 & 1.0 \\
Cobre & $0.038 \pm 0.0026$ & $0.080 \pm 0.0091$ & 0.000 & 0.42 & $\mathrm{Nd}$ & $\mathrm{Nd}$ \\
Hierro & $0.26 \pm 0.016$ & $0.31 \pm 0.018$ & 0.000 & 4.5 & $\mathrm{Nd}$ & $\mathrm{Nd}$ \\
Zinc & $15.0 \pm 0.95$ & $14.3 \pm 0.87$ & 0.016 & $\mathrm{Nd}$ & $\mathrm{Nd}$ & $\mathrm{Nd}$ \\
Manganeso & $0.0683 \pm 0.0163$ & $0.1582 \pm 0.0205$ & 0.000 & $\mathrm{Nd}$ & $\mathrm{Nd}$ & $\mathrm{Nd}$ \\
\hline
\end{tabular}

mientras que en la provincia de Huancané fue $\mathrm{Zn}>\mathrm{Fe}>\mathrm{Mn}>\mathrm{Cu}>\mathrm{Pb}>\mathrm{Cd}>\mathrm{Hg}$. $\mathrm{La}$ diferencia en la secuencia de concentración en algunos elementos químicos se debe a la existencia de minería aurífera artesanal de importante escala, siendo su principal impacto asociado con la evacuación de plomo (Loza del Carpio y Ccancapa, 2020) y otros metales pesados (Salas, 2009) a través de los ríos y riachuelos, los que finalmente drenan a través del río Ramis al lago Titicaca.

Se empleó el método espectrofotométrico utilizando la técnica de plasma de acoplamiento inductivo junto a un espectrofotómetro de emisión óptica ICP-OES (Roca et al., 2003; Silva et al., 2003), siguiendo el protocolo 7003 EPA 200.7 certificado por INACAL (LAS, 2021). Los datos fueron analizados mediante la prueba de comparación de medias, $\mathrm{t}$ Student $(\alpha=0.05)$, y contrastados con los referentes de los límites máximos permisibles de metales pesados en productos acuáticos (WHO/FAO, 2015; FSANZ, 2013; MHPRC, 2013).

\section{Resultados y Discusión}

La secuencia de las concentraciones medias de los metales pesados en las truchas arcoíris (Oncorhynchus mykiss) colectadas de la provincia de Puno fue: $\mathrm{Zn}>\mathrm{Fe}>\mathrm{Mn}>$ $\mathrm{Pb}>\mathrm{Cu}>\mathrm{Cd}>\mathrm{Hg}$, y en la provincia de Huancané: $\mathrm{Zn}>\mathrm{Fe}>\mathrm{Mn}>\mathrm{Cu}>\mathrm{Pb}>\mathrm{Cd}>$ $\mathrm{Hg}$ (Cuadro 2).

A nivel general, los niveles de metales pesados encontrados en los músculos de la trucha arcoíris están por debajo de los niveles máximos permisibles (WHO/FAO, 2015; FSANZ, 2013; MHPRC, 2013; OMS, 2016; Memet et al., 2017; European Commission, 2008), lo cual es un aspecto favorable, puesto que aún no constituye problema para la salud pública, pudiéndose consumir sin mucha preocupación (Mahjoub et al., 2020). El comparativo entre ambas zonas evidencia algunas variaciones $(p<0.05)$ en el contenido de los metales pesados, lo cual puede ser indicador de la diferencia en el contenido de estos contaminantes en los cuerpos de agua; 
sin embargo, todos los valores están por muy debajo de los niveles máximos permisibles.

El plomo es un elemento no esencial para los seres vivos y la exposición excesiva al plomo causa problemas neurológicos, alteraciones hematológicas, insuficiencia renal, hipertensión y cáncer (Bosch et al., 2016; Garcia et al., 2010; Shaheen et al., 2016). Las concentraciones medias de $\mathrm{Pb}$ en los músculos de la trucha en Puno $(0.051 \pm 0.0028$ mg.kg-1 $)$ y de Huancané $(0.049 \pm 0.0032$ mg. $\left.\mathrm{kg}^{-1}\right)$ son diferentes $(\mathrm{p}<0.05)$; sin embargo, estuvieron por debajo de los límites máximos permisibles para $\mathrm{Pb}$ en peces (Ministry of Health of the People's Republic of China [MHPRC, 2013]; Food Standards Australia and New Zealand [FSANZ, 2013]; World Health Organization / Food and Agriculture Organization of the United Nations [WHO/ FAO, 2015; FAO, 2003]).

La exposición excesiva al cadmio está asociada con efectos renales, pulmonares, hepáticos, esqueléticos, reproductivos y cáncer (Bosch et al., 2016; Shaheen et al., 2016). La concentración media de $\mathrm{Cd}$ en el músculo de la trucha fue menor a 0.022 mg. $\mathrm{kg}^{-1}$ (Cuadro 2), valor que se encuentra por debajo de los niveles máximos permisibles recomendados (MHPRC, 2013).

Los elementos como el $\mathrm{Cd}, \mathrm{Pb}$ y $\mathrm{Hg}$ son categorizados como elementos tóxicos, sin función metabólica; sin embargo, pueden ser perjudiciales para el humano, incluso en bajas concentraciones, cuando se ingieren durante periodos prolongados (Somers, 1974). En el presente estudio, las concentraciones de estos elementos en el músculo de la trucha no superaron los límites permisibles $(0.05 \mu \mathrm{g} / \mathrm{g} ; 0.3 \mu \mathrm{g} / \mathrm{g}$ y $0.5 \mu \mathrm{g} / \mathrm{g}$, respectivamente) para el consumo humano señalado por la Comisión Europea (2008).

$\mathrm{El} \mathrm{Fe}, \mathrm{Cu}, \mathrm{Mn}$ y $\mathrm{Zn}$ son elementos esenciales debido a su importante papel en los sistemas biológicos (Pis et al., 2008; Fallah et al., 2011; Bosch et al., 2016). En el presente estudio se encontró concentración $(\mathrm{p}<0.05)$ de $\mathrm{Fe}, \mathrm{Mn}$ y $\mathrm{Cu}$ en las truchas de Huancané en comparación con las de Puno, indicando que ese cuerpo de agua estaría acumulando mayores niveles de contaminantes, lo cual sería debido a que esa zona recibe la descarga del río Ramis (Cuadro 2). Se ha demostrado que los músculos oscuros contienen concentraciones de $\mathrm{Fe}$ más altas que los músculos claros (Lal, 1995), lo cual podría explicar que los tejidos de las truchas de crianza intensiva de la provincia de Huancané son más oscuros que los de la Provincia de Puno. En todo caso, las concentraciones obtenidas de $\mathrm{Fe}, \mathrm{Cu}, \mathrm{Mg}$ y $\mathrm{Zn}$ no superaron los límites permisibles (Alonso, 2019).

\section{Conclusiones}

- La secuencia de las concentraciones medias de los metales pesados en las truchas arcoíris (Oncorhynchus mykiss) colectadas de la provincia de Puno fue de $\mathrm{Zn}>\mathrm{Fe}>\mathrm{Mn}>\mathrm{Pb}>\mathrm{Cu}>\mathrm{Cd}>\mathrm{Hg}$ y en la provincia de Huancané fue de $\mathrm{Zn}>\mathrm{Fe}>\mathrm{Mn}>\mathrm{Cu}>\mathrm{Pb}>\mathrm{Cd}>\mathrm{Hg}$.

- Las concentraciones de los metales pesados evaluados en los músculos de las truchas arcoíris están por debajo de los límites máximos permisibles, siendo aptos para el consumo humano.

\section{Literatura Citada}

1. Afshan S, Ali S, Ameen US, Farid M, Bharwana SA, Hannan F, Ahmad R. 2014. Effect of different heavy metal pollution on fish effect of different heavy metal pollution on fish. Res J Chem Environ Sci 2: 74-79.

2. Ali H, Khan E, Ilahi I. 2019. Environmental chemistry and ecotoxicology of hazardous heavy metals: environmental persistence, toxicity, and bioaccumulation. J Chem-NY 2019: 6730305 doi: $10.1155 / 2019 / 6730305$ 
3. Alonso A. 2019. Metales pesados. Unión Europea. Contenidos máximos en metales pesados en productos alimenticios. Bogotá, Colombia. [Internet]. Disponible en: http://plaguicidas.comercio.es/MetalPesa.pdf

4. Aranibar MJ, Calmet E, Roque B. 2013. Valoración energética de nuevos alimentos para truchas arco iris (Oncorrynchus mykiss). Rev Investig Altoandina 15: 275-284. doi: 10.18271/ ria.2013.9

5. Authman MMN, Zaki MS, Khallaf EA, Abbas HH. 2015. Use of fish as bio-indicator of the effects of heavy metals pollution. J Aquac Res Dev 6: 328 . doi: 10.4172/2155-9546.1000328

6. Balami S, Sharma A, Karn R. 2019. Significance of nutritional value of fish for human health. Malaysian J Halal Res 2: 32-34. doi: 10.2478/mjhr-2019-0012

7. Bawuro AA, Voegborlo RB, Adimado AA. 2018. Bioaccumulation of heavy metals in some tissues of fish in lake Geriyo, Adamawa State, Nigeria. J Environ Public Health 2018: 1854892. doi: $10.1155 / 2018 / 1854892$

8. Bosch AC, O'Neill B, Sigge GO, Kerwath SE, Hoffman LC. 2016. Heavy metals in marine fish meat and consumer health: a review. J Sci Food Agric 96: 32-48. doi: 10.1002/jsfa.7360

9. Cáceres LF, Ramos OE, Valdez $S N$, Choque RR, Choque RG, et al. 2013. Fractionation of heavy metals and assessment of contamination of the sediments of Lake Titicaca. Environ Monit Assess 185: 9979-9994. doi: 10.1007/s10661-013-3306-0

10. Canli M, Atli G 2003. The relationships between heavy metal $(\mathrm{Cd}, \mathrm{Cr}, \mathrm{Cu}, \mathrm{Fe}$, $\mathrm{Pb}, \mathrm{Zn})$ levels and the size of six Mediterranean fish species. Environ Pollut 121: 129-136. doi: 10.1016/S02697491(02)00194-X

11. Chura R, Mollocondo H. 2009. Desarrollo de la acuicultura en el Lago Titicaca (Perú). AquaTIC 31: 6-19.
12. Doan HV, Jaturasitha S, Yamaka $S$, Faggio C, Pornsopin P. 2020. Proximate and nutritional content of rainbow trout (Oncorhynchus mykiss) flesh cultured in a tropical highland area. Braz Arch Biol Technol. 63: e20180234. doi: 10.1590/1678-4324-2020180234

13. Durmus M. 2019. Fish oil for human health: omega-3 fatty acid profiles of marine seafood species. Food Sci TechBrazil 39(Suppl 2): 454-461. doi: 10.1590/ fst. 21318

14. El-Moselhy KM, Othman AI, El-azem $H A$, Sea R. 2014. Bioaccumulation of heavy metals in some tissues of fish in the Red Sea, Egypt. J Basic Appl Zool 1: 97-105. doi: 10.1016/j.ejbas.2014.06.001

15. European Commission. 2008. Commission Regulation (EC) No. 629/2008 of 2 July 2008 amending Regulation (EC) No 1881/2006 setting maximum levels for certain contaminants infoodstuffs. Off J Europ Union 173/6.

16. Everett GV. 1973. The rainbow trout Salmo gairdneri (Rich) fishery of Lake Titicaca. J Fish Biol 5: 429-440. doi: 10.1111/j.1095-8649.1973.tb04472.x

17. Fallah AA, Saei SS, Nematollahi A, Jafari T. 2011. Comparative study of heavy metal and trace element accumulation in edible tissues of farmed and wild rainbow trout (Oncorhynchus mykiss) using ICP-OES technique. Microchem J 98: 275-279. doi: 10.1016/j.microc.2011.02.007

18. FAO. 2003. Legal Notice No 66/2003: Heavy metal regulations. Rome: FAO. [Internet]. Available in: http://extwprlegs 1.fao.org/docs/pdf/eri42405.pdf.

19. FAO. 2005. National Aquaculture Sector Overview - Peru. Rome: FAO. [Internet]. Available in: http://www.fao.org/fishery/countrysector/ naso peru/en.

20. [FSANZ] Food Standards Australia and New Zealand. 2013. Australia New Zealand Food Standards Code, 
Standard 1.4.1, Contaminants and Natural Toxicants. [Internet]. Available in: https://www.legislation.gov.au/Details/ F2013C00140

21. Gammons CH, Slotton DG, Gerbrandt B, Weight W, Young CA, McNearny $R L, C a ́ m a c ~ E, W$, et al. 2006. Mercury concentrations of fish, river water, and sediment in the Río Ramis - Lake Titicaca watershed, Peru. Sci Total Environ 368: 637-648. doi: 10.1016/ j.scitotenv.2005.09.076

22. Garcia J, Mendez J, Pasaro E, Laffon B. 2010. Genotoxic effects of lead: an updated review. Environ Int 36: 623-636. doi: 10.1016/j.envint.2010.04.011

23. Gündoddu A, Erdem M. 2008. The accumulation of heavy metals (cooper and zinc) in the tissues of rainbow trout (Onchorhyncus mykiss Walbaum, 1792). J Fisheries Sci 2: 41-50. doi: 10.3153/jfscom.2008004

24. Huseen HM, Mohammed AJ. 2019. Heavy metals causing toxicity in fishes. In: IOP Conf. Series: J Phys Conf Ser 1294: 062028. doi: 10.1088/1742-6596/ $1294 / 6 / 062028$

25. Kwasek K, Thorne-Lyman AL, Phillips M. 2020. Can human nutrition be improved through better fish feeding practices? a review paper. Crit Rev Food Sci 60: 3822-3835. doi: 10.1080/10408398.2019.1708698

26. Lal SP. 1995. Macro and trace elements in fish and shellfish. In: Ruiter A (ed). Fish and fishery products: composition, nutritive properties and stability. $\mathrm{CAB}$ International. p 187-214.

27. [LAS] Laboratorios Analíticos de Sur. 2021. Informes de ensayo. [Internet]. Disponible en: https://www.laboratoriosanaliticosdelsur.com/nosotros.php

28. Loza del Carpio AL, Ccancapa Y. 2020. Mercurio en un arroyo altoandino con alto impacto por minería aurífera artesanal (La Rinconada, Puno, Perú). Rev Int Contam Ambie 36: 33-44. doi: 10.20937/RICA.2020.36.53317
29. Mahjoub M, El Maadoudi M, Smiri Y. 2020. Metallic contamination of the muscles of three fish species from the Moulouya river (Lower Moulouya, Eastern Morocco). Int J Ecol 2020: 8824535. doi: $10.1155 / 2020 / 8824535$

30. Majlesi M, Malekzadeh J, Berizi E, Toori MA. 2019. Heavy metal content in farmed rainbow trout in relation to aquaculture area and feed pellets. Foods and Raw Materials 7: 329-338. doi: 10.21603/2308-4057-2019-2-329-338

31. Martínez I, Zuleta R, Pacheco A, Sanjines J. 2007. Cooperation on the Lake Titicaca. UNESCO Technical Documents in Hydrology No. 32. Paris. [Internet]. Available in: https://unesdoc.unesco.org/ark:/48223/pf0000153200

32. Maurya PK, Malik DS, Yadav KK, Kumar A, Kumar S, Kamyab H. 2019. Bioaccumulation and potential sources of heavy metal contamination in fi sh species in River Ganga basin: Possible human health risks evaluation. Toxicol Rep 6: 472-481. doi: 10.1016/j.toxrep.2019.05.012

33. Memet V, Gülderen KK, Alp A. 2017. Heavy metal and arsenic concentrations in rainbow trout (Oncorhynchus mykiss) farmed in a dam reservoir on the Firat (Euphrates) River: Risk-based consumption advisories. Sci Total Environ 600: 1288-1296. doi: 10.1016/j.scitotenv.2017.05.052

34. [MHPRC] Ministry of Health of the People's Republic of China. 2013. National food safety standard, maximum levels of contaminants in foods (GB27622012). [Internet]. Available in: https:// www.seafish.org/document $/$ ?id $=9 \mathrm{bc}-$ 28b9d-df1d-42cd-a098-32f71e8710c8

35. Mohanty BP, Ganguly S, Mahanty A, Mitra T, Patra S, Karunakaran D, et al. 2018. Fish in human health and nutrition. Adv Fish Res 7: 189-218.

36. Monroy M, Maceda-veiga A, Sostoa A. 2014. Metal concentration in water, sediment and four fish species from 
Lake Titicaca reveals a large-scale environmental concern. Sci Total Environ 487: 233-244. doi: 10.1016/j.scitotenv.2014.03.134

37. Moreno E, Pérez G, Alfaro R, Aparicio M, Atencio S, Goyzueta G 2017. Determinación interactiva de metales totales en las aguas de la bahía interior del. Rev Investig Altoandina 19: 125-134.

38. [OMS] Organización Mundial de la Salud. 2016. Norma general para los contaminantes y las toxinas presentes en los alimentos y piensos (Codex Stan No. 193). Internet]. Disponible en: http:// www.fao.org/fao-who-codexalimentarius/shproxy/en/? lnk=$1 \&$ url=https $\% 253 \mathrm{~A} \% 252 \mathrm{~F} \% 252$ Fworkspace.fao.org\%252Fsites\%$252 \mathrm{Fcodex} \% 2-52 \mathrm{FStandards} \%$ 252FCODEX\%2BSTAN\%2B193 1995\%252FCXS_193s.pdf

39. Onda Azul. 2020. Puno: Tramitan solicitudes para la exportación de más de 20 mil toneladas de trucha a Bolivia. Puno. [Internet]. Disponible en: https:// mercadosyregiones.com/2020/04/30/ puno-tramitan-solicitudes-para-laexportacion-de-mas-de-20-toneladas-detrucha-a-bolivia/

40. Peter S, Chopra S, Jacob JJ. 2013. A fish a day, keeps the cardiologist away! - A review of the effect of omega-3 fatty acids in the cardiovascular system. Indian J Endocrinol Metab 17: 422-429. doi: $10.4103 / 2230-8210.111630$

41. Pis MA, Lezcano MM, Serrano P. 2008. Metales pesados en trucha (Micropterus salmoides floridanus) de la presa Hanabanilla, Cuba. AquaTIC 29: 1-9.

42. Rajeshkumar S, Li X. 2018. Bioaccumulation of heavy metals in fish species from the Meiliang Bay, Taihu Lake, China. Toxicol Rep 5: 288-295. doi: 10.1016/ j.toxrep.2018.01.007

43. Roca P, Oliver J, Rodriguez A. 2003. Bioquímica: técnicas y métodos. Madrid, España: Ed Hélice. 347 p.
44. Salas FB. 2009. Determinación de metales pesados en las aguas del río Ananea debido a la actividad minera aurífera, Puno-Perú. Rev Investig Esc Posgrado 5(4): 47-53.

45. Salvarredy-Aranguren $M$, Probst A, Roulet M, Isaure MP. 2008. Contamination of surface waters by mining wastes in the Milluni Valley (Cordillera Real, Bolivia): mineralogical and hydrological influences. Appl Geochem 23: 1299-1324. doi: 10.1016/j.apgeochem.2007.11.019

46. Shaheen N, Ahmed MK, Islam MS, Habibullah-Al-Mamun M, Tukun AB, Islam S, M A Rahim AT. 2016. Health risk assessment of trace elements via dietary intake of 'non-piscine protein source' foodstuffs (meat, milk and egg) in Bangladesh. Environ Sci Pollut Res Int 23: 7794-7806. doi: 10.1007/s11356015-6013-2

47. Silva JCJ, Baccan N, Nóbrega JA. 2003. Analytical performance of an inductively coupled plasma optical emission spectrometry with dual view configuration. J Braz Chem Soc 14: 210315. doi: 10.1590/s0103-50532003000400026

48. Somers E. 1974. The toxic potential of trace metals in foods. A review. J Food Sci 39: 215-217.

49. Thilsted SH, Thorne-lyman A, Webb P, Bogard JR, Subasinghe R, Phillips MJ, Allison EH. 2016. Sustaining healthy diets: the role of capture fisheries and aquaculture for improving nutrition in the post-2015 era. Food Policy 61: 126131. doi: 10.1016/j.foodpol.2016.02.005

50. [WHO/FAO] World Health Organization / Food and Agriculture Organization of the United Nations. 2015. Codex Alimentarius Commission, General Standard for Contaminants and Toxins in Food and Feed (CODEX STAN No. 193-1995). [Internet]. Available in: http://refhub.elsevier.com/S00489697(17)31152-X/rf0365 
51. Wu W, Wu P, Yang F, Sun DL, Zhang DX, Zhou YK. 2018. Assessment of heavy metal pollution and human health risks in urban soils around an electronics manufacturing facility. Sci Total Environ 630: 53-61. doi: 10.1016/j.scitotenv.2018.02.183
52. Zhao S, Feng C, Quan W, Chen X, Niu J, Shen Z. 2012. Role of living environments in the accumulation characteristics of heavy metals in fishes and crabs in the Yangtze River Estuary, China. Mar Pollut Bull 64: 1163-1171. doi: 10.1016/ j.marpolbul.2012.03.023 